\title{
Corela
}

Cognition, représentation, langage

7-2| 2009

Vol. $7, \mathrm{n}^{\circ} 2$

\section{Compte-rendu de lecture}

Le Regard dans la langue des signes: Anaphore en langue des signes française de Belgique (LSFB) : morphologie, syntaxe, énonciation, par Laurence Meurant

\section{Annie Risler}

\section{OpenEdition}

\section{Journals}

Édition électronique

URL : http://journals.openedition.org/corela/210

DOI : $10.4000 /$ corela. 210

ISSN : 1638-573X

\section{Éditeur}

Cercle linguistique du Centre et de l'Ouest - CerLICO

\section{Référence électronique}

Annie Risler, « Compte-rendu de lecture », Corela [En ligne], 7-2 | 2009, mis en ligne le 15 décembre 2009, consulté le 22 septembre 2020. URL : http://journals.openedition.org/corela/210 ; DOI : https:// doi.org/10.4000/corela.210

Ce document a été généré automatiquement le 22 septembre 2020.

\section{(c) (i) (2)(2)}

Corela - cognition, représentation, langage est mis à disposition selon les termes de la licence Creative Commons Attribution - Pas d'Utilisation Commerciale - Partage dans les Mêmes Conditions 4.0 International. 


\section{Compte-rendu de lecture}

Le Regard dans la langue des signes: Anaphore en langue des signes française de Belgique (LSFB) : morphologie, syntaxe, énonciation, par Laurence Meurant

\section{Annie Risler}

\section{RÉFÉRENCE}

Coll. Rivages Linguistiques, Presses Universitaires de Rennes, 2008, 290 p. En

collaboration avec les Presses Universitaires de Namur.

1 Laurence Meurant démontre dans cet ouvrage que la prise en compte du regard apparaît comme un point d'appui solide et heuristique pour la mise en évidence de l'organisation grammaticale de la Langue des Signes Française de Belgique (LSFB) et de toute langue signée. Pour cela, elle s'est appuyée sur un modèle général de description du langage, la glossologie, qu'elle met à l'épreuve des spécificités engendrées par la modalité visuo-gestuelle d'une langue signée.

2 Cet ouvrage s'adresse donc aussi bien aux non signeurs qu'aux signeurs. Les premiers pourront accéder au fonctionnement d'une langue qui a comme particularité d'utiliser l'espace, et d'être produite par des articulateurs multiples (membres supérieurs, buste, regard, face) qui autorisent à la fois la successivité et la simultanéité des unités linguistiques. Les seconds pourront se livrer à une étude comparative entre leur LS et la LSFB, voir envisager sous un jour nouveau le fonctionnement de leur LS à partir des outils développés.

3 Le regard n'a pas de valeur morphologique en soi, mais associé à d'autres fragments de signifiant, comme les mouvements manuels, il instaure des valeurs différentielles de signifié. Le signeur peut, alternativement, regarder son interlocuteur dans un regard adressé, ou couper cette adresse pour porter son regard sur ses mains, sur une portion de l'espace ou se perdre dans un regard vague. Ces différentes valeurs du regard conjuguées avec des paramètres manuels instaurent des oppositions qui déterminent différentes catégories morphologiques qui seront présentées tout au long du livre. 
4 Cette étude se démarque des travaux antérieurs, du fait des positions d'analyse adoptées, et de leurs conséquences sur les descriptions et catégorisations auxquelles on arrive. L'auteur revendique deux points d'originalité: le fait de prendre en considération la direction du regard du signeur, et celui de ne pas recourir au sens pour expliquer la forme. Un articulateur parmi d'autres, le regard a longtemps été peu considéré dans les études sur les langues signées dans lesquelles l'étude des mouvements manuels était survalorisée. De la même manière, l'iconicité propre aux LS et la prégnance du corps en mouvement ont incité à lier très fortement la forme et le sens. Un troisième préalable, que l'auteur ne pointe pas spécialement tant il lui parait évident, va cependant faire la distinction avec les études précédentes. Elle rappelle à plusieurs reprises le caractère abstrait des unités linguistiques, qui comportent une ou plusieurs valeurs de signifié, qu'on peut reconstituer à partir de l'analyse des mouvements produits par les différents articulateurs, ou signifiants, mais qui ne se confondent pas avec la matérialité des gestes. Par le fait de s'abstraire de la matérialité si évidente d'une langue motivée, Laurence Meurant se démarque clairement des analyses qui expliquent la forme gestuelle par un recours au sens et ne peuvent du coup rendre compte des phénomènes proprement morphologiques, syntaxiques et pragmatiques. Cette position théorique et méthodologique l'amène à discuter et redéfinir la plupart des concepts employés dans les travaux sur les langues signées : les locus, les transferts, les classificateurs, les catégories de verbes.

5 Pour cela, elle est soutenue par son cadre théorique, celui de la glossologie. Ce volet de la Théorie de la Médiation, développée par Gagnepain, s'attaque au «fonctionnement grammatical du dire ». Ce modèle linguistique construit pour les langues orales est assez généraliste pour guider l'étude d'une langue très différente. Le mot y est défini selon un critère d'autonomie formelle et non d'emploi, envisagé comme une unité constituée de la solidarité de valeurs enclitiques. La complexité interne d'un mot résulte de la coprésence de plusieurs fragments enclitiques associés au radical autonome. Conséquemment, la morphologie renvoie à l'étude de la variation interne au mot, donc à l'étude des valeurs possibles de ces fragments enclitiques selon un système d'oppositions, et la syntaxe concerne l'étude des rapports réciproques entre les mots, ou étude des contraintes communes et bilatérales qui réduisent l'autonomie des constituant complémentaires.

6 Cette notion de mot appliquée à une langue signée n'est pas donnée d'emblée. Ainsi les exemples donnés dans le livre portent la marque de l'analyse effectuée par le linguiste, qui produit un double découpage du flux gestuel : en unités de sens juxtaposées, ellesmêmes décomposables en un ou plusieurs morphèmes.

7 Signalons ici que les exemples sont présentés de manière à être compris par tous les lecteurs, quelle que soit leur connaissance de la LSFB : chaque unité est associée à une photo sous laquelle sont indiquées d'une part la direction du regard et d'autre part les valeurs grammaticales des unités manuelles. La traduction en français qui figure en dessous est le plus littérale possible. Tous les exemples sont présentés également sous forme vidéo, bien qu'il ne soit pas indispensable de recourir à la forme signifiante pour suivre l'analyse grammaticale proposée.

8 Le livre est composé de trois parties qui s'appellent l'une l'autre :

9 La première partie fait rentrer dans le vif du sujet, à savoir le fonctionnement morphologique et syntaxique de la LSFB, en illustrant en quoi le regard constitue un 
point d'entrée pertinent dans l'analyse à partir d'un exemple particulier : les signes de pointage.

10 La deuxième partie rassemble des éléments de morphologie puis de syntaxe, en accord avec le cadre théorique posé.

11 Puis la troisième partie développe une réflexion sur des structures syntaxiques anaphoriques, et sur la notion pragmatique de point de vue. Ce qui permet de revenir au point de départ: la nécessité de prendre en compte la direction du regard pour analyser les productions signées.

12 Ce parcours a l'avantage de très clairement distinguer les différents niveaux d'analyse de la langue, qui reposent toujours sur des critères strictement formels. C'est là une des grandes qualités de l'ouvrage. Les mêmes exemples sont repris dans les différents chapitres, ce qui renforce la cohérence de l'ensemble.

13 La première partie est intitulée : le regard, de la déixis à l'anaphore. L'auteur présente une étude du système des pronoms personnels en LSFB qui montre que le regard porté sur l'interlocuteur constitue une véritable fonction d'adresse et à ce titre correspond à un indice d'énonciation. Les références déictiques comme les valeurs de personnes, qui sont produites par des pointages vers les protagonistes de la situation d'énonciation, sont toujours accompagnées d'un regard adressé à l'interlocuteur. A partir de ce constat, l'auteur montre de manière distinctive comment la mise en suspend de l'adresse du regard provoque une mise en suspend de l'énonciation et une grammaticalisation de l'espace.

14 Les langues signées utilisent l'espace à des fins de référence: pour désigner une personne ou une entité, le signeur lui établit un emplacement dans l'espace auquel il peut recourir dans la suite de son discours. Cet emplacement peut être désigné manuellement, au moyen d'un pointé (index tendu dirigé vers cet emplacement). Laurence Meurant montre que la direction du regard met en effet en évidence au plan formel une opposition entre les plans de l'anaphore et de la déixis : un pointage manuel vers un emplacement correspond une valeur déictique de $3^{\text {ème }}$ personne, s'il est accompagné d'un regard adressé à l'interlocuteur; alors qu'il instaure une valeur anaphorique de locus si le regard est porté sur l'emplacement désigné. Ces procédés anaphoriques mis en jeu par la suspension de l'adresse du regard sont qualifiés d'anaphore pseudo-déictique puisqu'ils s'appuient sur des gestes de désignation spatiale.

15 La direction du regard établit également une distinction concernant la place du signeur, entre énonciation et énoncé: le buste du signeur occupe une position énonciative, lorsque le regard est adressé ; il prend une valeur anaphorique quand le regard est suspendu. Ce procédé traditionnellement décrit comme un transfert personnel est ici analysé comme une neutralisation de la valeur de personne attachée au buste du signeur. A partir de là, l'auteur montre comment le corps du signeur peut déterminer une origine à un nouveau système de références personnelles hors du cadre de l'énonciation, dans le discours rapporté, par le jeu du regard adressé à un interlocuteur associé à un locus.

16 Cette première partie constitue une belle entrée en matière : à partir de la question de la différence entre anaphore et déixis, dans laquelle la direction du regard du locuteur constitue un paramètre distinctif, l'auteur redéfinit les catégories grammaticales fondamentales de locus et de transfert. 

question le classement traditionnel entre des verbes neutres (invariables), verbes à classificateurs de position ou de déplacement (définis sémantiquement, et caractérisés par une configuration manuelle et une orientation variables) et verbes directionnels (orientables entre sujet et objet). La classification proposée par l'auteur se fonde uniquement sur des critères formels. En particulier, la catégorie des verbes de position est supprimée. L'existence est exprimée par la copule enclitique, considérée comme un fragment du nom. Il s'agit d'une nouvelle catégorie morphologique, dont la valeur est marquée par un mouvement d'arrêt qui porte sur le radical, sur un classificateur ou une particule locative.

évidence le comportement solidaire du radical des différentes catégories morphologiques, sont ensuite repris dans chapitre sur la syntaxe pour objectiver le comportement distinctif des noms et des différentes catégories de verbes, dans des phénomènes d'accord et de rection.

26 L'auteur détermine ainsi les contraintes mutuelles qui restreignent l'autonomie des constituants complémentaires, dans des relations comme: le pluriel défini (entre plusieurs mots verbaux ou plusieurs mots nominaux), la relation verbe-complément spatialisé, le syntagme de localisation (entre deux mots nominaux), le syntagme épithète (entre deux mots nominaux), ou le syntagme nom-transfert personnel. 

de distinguer plusieurs espaces, les effets de la simultanéité des articulateurs sur l'expression des morphèmes discontinus, l'opposition verbo-nominale, les catégories de verbes et la distinction entre transfert et discours rapporté.

- La quadripartition de l'espace : l'espace physique se découpe en 4 espaces structurés par un comportement particulier du regard

- l'espace déictique, des valeurs de personnes, regard adressé ;

- l'espace frontal, des valeurs de locus, regard sur le locus ;

- l'espace du signeur et l'espace latéral, organisés en fonction de la valeur du corps du signeur donnée par le transfert personnel, regard non adressé, hors espace frontal.

- Simultanéité et linéarité : la simultanéité et la linéarité matérielles du geste ne sont que trompe l'œil, et doivent être distinguées du caractère imbriqué ou discontinu des éléments de signifiant. La valeur discontinue s'exprime selon trois positions : l'anté-position, la postposition, ou la symposition. Dans ce dernier cas, il y a articulation conjointe mais par deux articulateurs distincts.

- Opposition verbo-nominale : cette opposition se joue sur des valeurs exclues d'un type ou l'autre : le type nominal est incompatible avec la valeur d'accompli, alors que le type verbal est incompatible avec la valeur de copule enclitique.

- -Classification des verbes : la classification proposée reconnaît 4 groupes de verbes, à partir de l'étude des loci : nombre de loci, imbriqués au radical ou discontinus, et influence ou non de la neutralisation de personne sur les loci. L'étude des variations de classificateurs détermine des sous catégories dans les groupes 3 et 4 , entre des verbes qui admettent des classificateurs et d'autres pas.

- Groupe 1:2 valeurs de locus imbriquées au radical, l'un étant contraint en cas de neutralisation de personne ; sans choix de classificateur

- Groupe $2: 2$ valeurs de locus imbriquées au radical, non influencés par la neutralisation de personne, avec des choix de classificateurs

- Groupe 3 : deux valeurs de locus répartis de manière discontinue

- Groupe 4 : une seule valeur de locus.

- Dialogue rapporté et transfert personnel : l'étude du regard montre qu'il est nécessaire de distinguer ces deux phénomènes, souvent confondus dans la notion de transfert personnel 
telle qu'elle a été décrite par Cuxac. En dialogue rapporté, le regard du locuteur installe face à lui un interlocuteur imaginaire, le regard a lors une fonction indicielle. La prise de rôle ouvre un second espace déictique face au signeur. Alors que dans le transfert personnel, qui est marqué par une neutralisation de la valeur de personne, le regard représente la perception d'une instance sans parole et active l'espace du signeur et l'espace latéral.

31 Au long de son exposé, Laurence Meurant va interroger successivement tous les termes de la linguistique, au premier rang desquels ceux de nom, verbe et adjectif. Elle s'interdit d'utiliser telle catégorie grammaticale communément admise avant de s'être assurée qu'elle est pertinente pour la langue étudiée. Le lecteur peut suivre pas à pas l'élaboration $\mathrm{du}$ système descriptif, qui commence par discuter les définitions traditionnelles des catégories identifiées dans d'autres cadres linguistiques pour les LS, comme les transferts, les pronoms, les locus ou les classificateurs. Ces catégories sont ensuite redéfinies au niveau strictement formel, ce qui amène des ajustements parfois importants, comme dans le cas des transferts, des verbes ou des classificateurs.

L'auteur a cependant choisi de conserver cette terminologie. C'est un parti pris qu'elle revendique, mais dont on peut se demander s'il est judicieux, dans un domaine où le manque de consensus amène les différents chercheurs à réutiliser les mêmes termes dans des contextes très différents. De nouvelles catégories sont mises en lumière, comme les indices de transfert personnel ou les copules enclitiques. Un glossaire, bienvenu, rappelle en fin de livre les définitions adoptées.

$33 \mathrm{Au}$ final, son étude sur la LSFB appelle à un réexamen de toutes les descriptions grammaticales des langues signées, en accordant une place centrale au regard, mais surtout en procédant à une analyse rigoureusement formelle. Laurence Meurant propose ici un ouvrage qui fera date dans les travaux de linguistique des langues signées.

\section{AUTEURS}

\section{ANNIE RISLER}

UMR 8163 STL, Université Lille 3 